\title{
Evolving Plant Diagnostics During a Pandemic
}

\author{
Alicyn Smart, ${ }^{1}$ Jan Byrne, ${ }^{2}$ Ray Hammerschmidt, ${ }^{2}$ Karen L. Snover-Clift, ${ }^{3}$ James P. Stack, ${ }^{4}$ Tania Brenes-Arguedas, ${ }^{5}$ Jeffrey B. Jones, ${ }^{6}$ \\ and Carrie L. Harmon ${ }^{6, t}$ \\ ${ }^{1}$ Cooperative Extension, University of Maine, Bangor, ME 04401 \\ ${ }^{2}$ Department of Plant, Soil and Microbial Sciences, Michigan State University, East Lansing, Ml 48824 \\ ${ }^{3}$ Cooperative Extension, Cornell, Ithaca, NY 14853 \\ ${ }^{4}$ Department of Plant Pathology, Kansas State University, Manhattan, KS 66506 \\ ${ }^{5}$ Department of Plant Pathology, University of California, Davis, CA 95616 \\ ${ }^{6}$ Department of Plant Pathology, University of Florida, Gainesville, FL 32611
}

Accepted for publication 30 November 2020.

\section{Abstract}

The National Plant Diagnostic Network (NPDN), comprising diagnostic professionals from more than 70 pathology, entomology, and nematology laboratories, safeguards U.S. plant systems through accurate diagnosis and effective communications with clients, partners, and stakeholders. As a USDA-NIFA extension program built on the land-grant university system, the network has dual responsibilities to extension clientele such as farmers and the green industry, as well as state and federal regulatory agencies. Following strategic planning in 2019, the network emerged with a concise plan and strong committees of network participants to enhance and sustain service to NPDN clientele and partners, even through significant disruptions like the 2020 coronavirus pandemic. The commitment to building diagnostic capacity and expertise across the country allows these plant clinics to assist during a response to detections of highconsequence plant pathogens by clearing healthy plants for commerce while identifying potential positives for regulators to quarantine and/or eradicate, similar to the test and trace efforts for human diseases such as COVID-19. In this review, we describe the network's recent activities to protect U.S. plant agriculture and natural ecosystems and its plans to improve and expand capacity for national plant biosecurity.

Keywords: diagnostics, biosecurity, plant pathology, extension
The National Plant Diagnostic Network (NPDN) has evolved since its inception in 2002, developing connections among and building capacity within 79 active pathology, entomology, and nematology laboratories in all U.S. states and territories (Fig. 1) (Stack et al. 2006). NPDN continues to support excellence in diagnostics as well as the generation of data that serve domestic and international plant protection and biosecurity efforts (Stack et al. 2014). As a critical component of the U.S. Department of Agriculture, National Institute of Food and Agriculture (USDA-NIFA) tactical sciences program (Anonymous 2017), the NPDN continues to innovate and contribute to surveillance and early detection of high-consequence pests and diseases via the timely deployment of technologies and human network resources. The current global response to COVID-19 has provided new insight into how we can improve plant problem detection, diagnostics, management, and communications through global surveillance systems (CarvajalYepes et al. 2019). The flexible, national scope of the NPDN model has tremendous advantages during unpredictable disease outbreaks.

\section{${ }^{\dagger}$ Corresponding author: C. L. Harmon; clharmon@ufl.edu}

Funding: This work was funded by University of Maine Cooperative Extension faculty startup funding.

The author(s) declare no conflict of interest.

(C) 2021 The American Phytopathological Society

\section{One Health and NPDN}

As the organization approaches the end of its second decade serving the agriculture and plant science communities, NPDN has renewed its focus on supporting diagnosticians and encouraging partnerships in the evolving landscape of plant pests. In early 2020,

\section{NPDN Lab Type by Discipline}

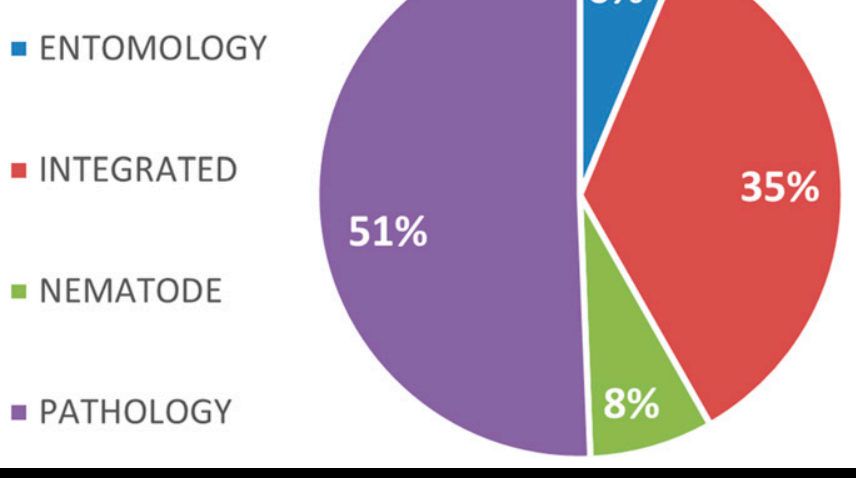

FIGURE 1

NPDN laboratories by type $(n=79)$. Integrated denotes laboratories with multiple functions, usually pathology plus entomology and/or nematology and/or weed science. 
the NPDN completed strategic planning, overhauling committees, and fine-tuning goals to focus resources on diagnostic efforts for the next decade. Eleven NPDN functional area committees composed of diagnostic personnel from across the United States are engaged in renewing and reinventing NPDN in support of the strategic plan (Anonymous 2019). These committees engage diagnostic professionals among agencies on the plant protection continuum to validate, translate, and disseminate protocols adapted from human, animal, and plant research (Fig. 2). NPDN is building the capacity of the laboratories and diagnosticians by providing more opportunities for knowledge transfer, from virtual onboard training for new diagnosticians to expanding the number of hands-on expertisebuilding workshops led by NPDN and Animal Plant Health Inspection Service (APHIS) specialists. These trainings began in 2003 and have grown from one workshop per year to up to 10 . The workshops have covered more than 30 topics including bacterial taxonomy, Phytophthora ramorum, bioinformatics, and accreditation. New topics entice repeat attendees, and introductory topics are renewed regularly to build the expertise of new diagnosticians, all to build a well-trained cohort nationwide. This incredible teamwork benefits global plant protection efforts as well as local disease and pest detection and management in a format that can be adapted to any system in the One Health concept (Mackenzie and Jeggo 2019). The convergence of One Health with innovations in detection technologies and NPDN's focus on virtual delivery of training, accreditation, and reporting is especially relevant during a year of pandemic stay-at-home orders and disease detection efforts (Wilkes et al. 2019).

\section{Partnerships for Training and Capacity}

Successful partnerships with state and federal agencies have resulted in technology transfer and diversified diagnostic capacity across the network. This is exemplified in NPDN participation in the APHIS National Plant Protection Laboratory Accreditation Program (NPPLAP), which was developed after a $P$. ramorum surge event in 2004 when 3,400 samples went to the single APHIS confirmatory laboratory in a few weeks, creating a months-long backlog (P. Shiel, personal communication). NPDN and regulatory partners now work together to prepare for and respond to sample surges of regulated pathogens such as $P$. ramorum, the causal agent of sudden oak death and ramorum blight diseases. The entire sampling-to-diagnosis process for $P$. ramorum is coordinated nationally by an NPDN designee and an APHIS program manager, ensuring samples can be tested quickly to enable appropriate response. To gain certification to run the specific triple quantitative polymerase chain reaction (qPCR) test for P. ramorum, individuals must pass an annual proficiency panel through the NPPLAP. Due to the specialized equipment and training required, only about a dozen

\section{Functional Areas}

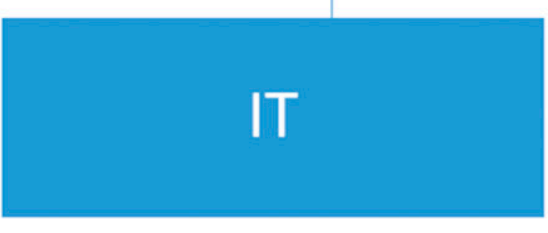

National Database Committee

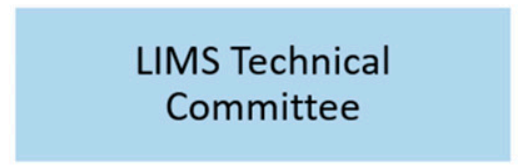

Data Use Committee
Diagnostics

\section{Professional Development Committee}

Accreditation Committee

\section{Proficiency Committee}

Protocols \& Validation Committee

\section{Communication}

\section{Online Communications \&}

Web Portal Committee

\section{Regulatory Relations Committee}

Outreach \& Extension Committee

FIGURE 2

NPDN strategic committee structure by functional area. 
laboratories maintain this certification across the United States in any given year.

During a $P$. ramorum surge, regulatory plant inspectors collect representative samples from blocks of plants at nurseries and retail outlets, which can then be funneled to their state's NPDN laboratory for genus-level triage using a serological test. All the plants in a block are held from sale pending results of triage testing. Samples positive for Phytophthora sp. are then funneled to regional laboratories for $P$. ramorum $\mathrm{qPCR}$ testing. If samples from new locations or hosts test positive for $P$. ramorum in these laboratories, they are forwarded to the APHIS confirmatory laboratory, where regulatory confirmation includes further molecular diagnostics and can result in regulatory action to protect the nursery industry and natural ecosystems. This robust and flexible "funnel and filter" model (Fig. 3) clears healthy plant material for commerce while catching potentially infected material to be confirmed by regulatory authorities, enabling states to release plant material for sale, reducing the workload on APHIS's confirmatory testing service, and preventing spread of the pathogen from nurseries to the landscape or environment (Stack et al. 2014).

This system was successfully deployed throughout 2019 and 2020, when $P$. ramorum was detected in multiple states (El-Lissy

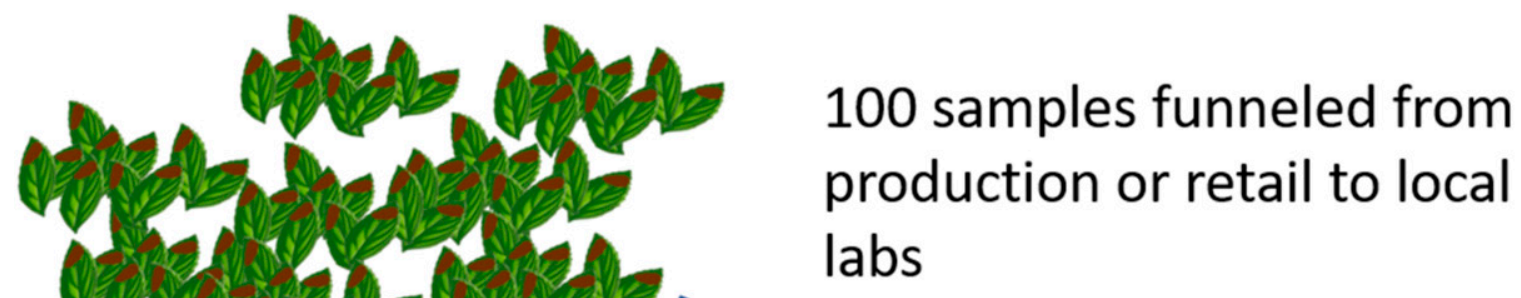

Serological triage at NPDN labs filter out samples negative for Phytophthora spp.

qPCR testing at NPDN labs provides initial identification of $P$. ramorum positive samples

\title{
production or retail to local
}
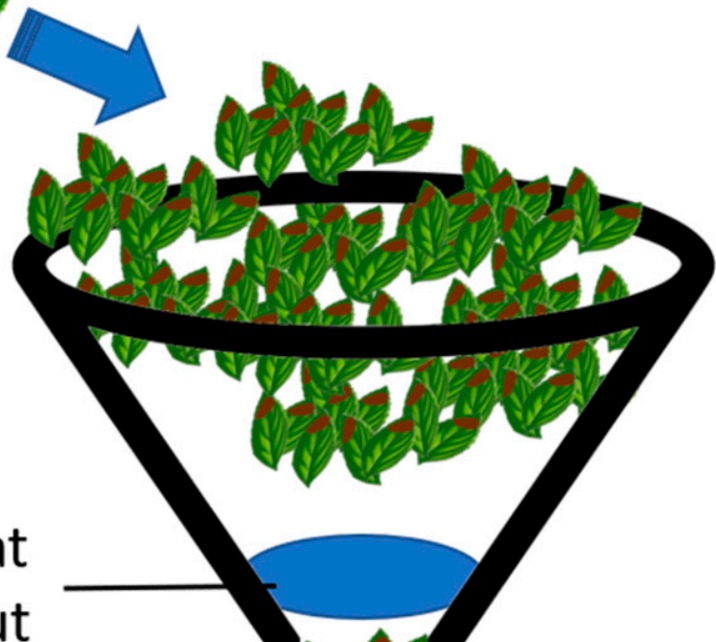

\author{
10 suspect \\ Phytophthora sp. \\ positive/ inconclusive \\ samples funneled to \\ expert-level qPCR labs
} Phytoph $\longrightarrow$

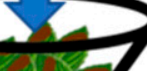
年

Forward 3 P. ramorum suspect positive/ inconclusive samples to APHIS confirmatory labs

FIGURE 3

The funnel-and-filter system of the NPDN triage program for Phytophthora ramorum samples. 
2019). State agricultural inspectors collected thousands of delimitation survey samples at wholesale and retail locations identified by tracing. More than a dozen NPDN laboratories triaged samples at the state level with genus-level enzyme-linked immunosorbent assay testing, quickly clearing thousands of negative plant samples and facilitating the release of nursery and retail stock for sale. Just $10 \%$ of those samples required qPCR at three certified NPDN regional center laboratories and the Pennsylvania Department of Agriculture laboratory. The funnel-and-filter system meant only 3\% of the total samples were forwarded to the two APHIS laboratories for regulatory confirmation, well within their capacity at just $10 \%$ of the surge that overwhelmed the laboratory in 2004. Remarkably, this system continued to operate seamlessly in spring and summer 2020, during the unprecedented COVID-19 disruptions, demonstrating the flexible nature of the network as sample capacity was coordinated at the national level to accommodate laboratory closures, shipping changes, and other COVID-related disruptions. The retail impact of this funnel-and-filter system from January 1, 2019, to November 1, 2020, is estimated conservatively at 11,350 plant samples collected by inspectors and funneled to NPDN laboratories, where each sample represents a block of at least three plants in a nursery or retail setting, with a retail value of $\$ 30 /$ plant, for a total retail value of US\$1,021,500. The estimated retail value of the plants cleared for commerce is US\$990,855 (97\%).

\section{Data In and Reporting Out}

NPDN laboratories upload a subset of all diagnostic records into the NPDN National Data Repository (NDR) at Purdue University's Center for Environmental and Regulatory Information Systems.
The NDR has been expanded to include critical information such as the methods used to confirm the diagnosis and the confidence in identification to support routine diagnosis, preparation for sample surge, and projects with partners (Fig. 4). New tools capture information to better serve collaborators, who utilize the diagnostic data for projects in epidemiology, pest risk analysis, climate change, and diagnostic emergency response (Frank and Just 2020; Klingeman et al. 2020; Narouei-Khandan et al. 2017). For example, Klingeman et al. (2020) analyzed thousands of scale insect records to indicate prevalence on ornamentals across the United States, aiding future documentation of movement and even climate change. Additionally, analysis of NDR data trends allows the network to prepare for new waves of samples and determine where to invest in infrastructure and training (Fig. 4).

\section{Adopting New Technologies and Pursuing New Directions}

The NPDN has partnered with NIFA and APHIS to outfit the laboratories with appropriate and vetted equipment and protocols in response to national needs for new diagnostic technologies and tests. In support of the NPPLAP certification administered by APHIS, planned deviation research in NPDN laboratories has expanded the number of instruments validated for regulatory protocols (Bush et al. 2016; Mavrodieva et al. 2017), and in December 2020 the Protocols and Validation Committee deployed a ring-test protocol to set a standard for method validation in the NPDN. Communications among NPDN and APHIS regarding anomalous results with new hosts, tests, or pathogens encourage the development of robust regulatory tests for deployment across the network, as well as building trusted relationships between the organizations.

\section{Total Phytophthoraspp. testing by NPDN labs}

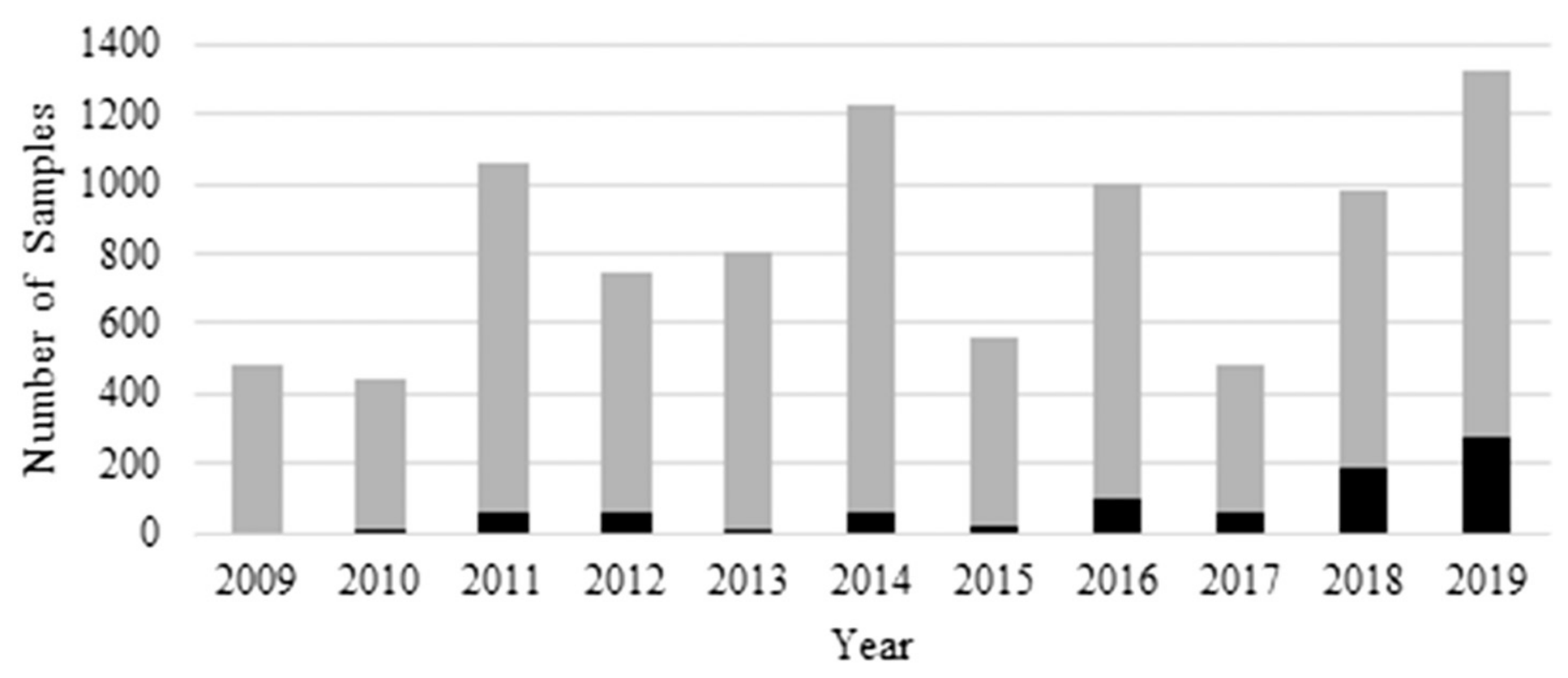

-Samples forwarded to APHS $\square$ Samples cleared by NPDN Labs

FIGURE 4

Total Phytophthora spp. testing performed by NPDN laboratories from 2009 through 2019 as part of the national Phytophthora ramorum program. Black bars represent the proportion of total samples that were forwarded to APHIS confirmatory laboratories. In 2004, before NPDN was engaged, 3,400 samples all went to the Beltsville laboratory. 
A memorandum of understanding and communications protocol drafted by the new NPDN Regulatory Relations Committee formalizes the relationship and communication between NPDN laboratories and their state regulatory counterparts. The importance of these relationships was demonstrated during the identification of Ralstonia solanacearum race 3 biovar 2 in geranium cuttings imported into a Michigan greenhouse in April 2020 (Anonymous 2020; Calabro et al. 2020). An NPDN diagnostician's quick diagnosis and timely communication with the Michigan state department of agriculture allowed the regulatory authorities to initiate coordinated state and federal agency responses in the 39 states that imported cuttings from the same facility. This included plant tracing, onsite testing in multiple states, and eradication of a pathogen with severe implications for U.S. agriculture.

NPDN is strategically allocating funding specifically to development of protocols, partnerships, and information technology solutions to improve the science of diagnostics and document impact of the network for its members and partners. The strong partnerships and effective coordination among agencies and service to extension clientele fostered by NPDN will continue to safeguard U.S. agriculture and natural systems into the next decade and beyond.

\section{Acknowledgments}

The authors acknowledge the reviewers, the National Plant Diagnostic Network USDA-NIFA cooperative agreements (2016-37620-25765, 201637620-25766, 2016-37620-25850, 2016-37620-25851, and 2016-37620-25852), and the data used with permission of the NPDN National Data Repository.

\section{Literature Cited}

Anonymous. 2017. Tactical Sciences to Protect the U.S. Agriculture Enterprise. Building Awareness and Developing a Coordinated Strategy. U.S. Department of Agriculture, Washington, DC. https://nifa.usda.gov/sites/default/ files/resource/Tactical-Sciences-report-on-conversation.pdf

Anonymous. 2019. NPDN Strategic Plan. https://www.npdn.org/system/files/ NPDN_Strategic_Plan-FINAL_20191005.pdf

Anonymous. 2020. USDA confirms Ralstonia solanacearum race 3 biovar 2 in geranium plants in greenhouse. https://www.aphis.usda.gov/aphis/newsroom/ stakeholder-info/sa_by_date/sa-2020/sa-04/ralstonia-greenhouse-geraniums.
Bush, E. A., Dennis, G., Mavrodieva, V., and Hansen, M. A. 2016. A planned deviation of a CPHST-validated protocol allows for use of a highthroughput real-time multicycler for detection of Phytophthora ramorum. Poster. National Plant Diagnostic Network 4th National Meeting: Advancing Diagnostics to Meet Plant Health Needs. https://conference. ifas.ufl.edu/npdn/Docs/Poster\%20Presentations/Bush\%20-\%20PLANNED\% 20DEVIATION\%20OF\%20A\%20CPHST-VALIDATED\%20PROTOCOL. pdf

Calabro, J., Allen, C., Royer, M., and Klopmeyer, M. 2020. Ralstonia webinar: What you need to know. https://www.hriresearch.org/ralstonia-webinarwhat-you-need-to-know.

Carvajal-Yepes, M., Cardwell, K., Nelson, A., Garrett, K., Giovani, B., Saunders, D. G. O., Kamoun, S., Legg, J. P., Verdier, V., Lessel, J., Neher, R.A., Day, R., Pardey, P., Gullino, M. L., Records, A. R., Bextine, B., Leach, J. E., Staiger, S., and Tohme, J. 2019. A global surveillance system for crop diseases. Science 364:1237-1239.

El-Lissy, O. 2019. APHIS confirms detection of Phytophthora ramorum-infected plants in commerce. https://content.govdelivery.com/accounts/USDAAPHIS/ bulletins/2509f86.

Frank, S. D., and Just, M. G. 2020. Can cities activate sleeper species and predict future forest pests? A case study of scale insects. Insects 11:142.

Klingeman, W. E., Chong, J.-H., Harmon, C. L., Ames, L., LeBude, A. V., and Chandran, P. 2020. Scale insect records from ornamental plants help to prioritize plant health resource development. Plant Health Prog. 21:278-287.

Mackenzie, J. S., and Jeggo, M. 2019. The One Health approach-Why is it so important? Trop. Med. Infect. Dis. 4:88.

Mavrodieva, V. A., Dennis, G., and Shiel, P. J. 2017. USDA APHIS NPPLAP proficiency testing and planned methods deviation as a part of the methods' validation process for a network of laboratories. Session 27-S. https:// www.apsnet.org/Documents/2017APS-ProgBk-Online.pdf.

Narouei-Khandan, H.A., Harmon, C. L., Harmon, P., Olmstead, J., Zelenev, V. V., van der Werf, W., Worner, S. P., Senay, S. D., and van Bruggen, A. H. C. 2017. Potential global and regional geographic distribution of Phomopsis vaccinii on Vaccinium species projected by two species distribution models. Eur. J. Plant Pathol. 148:919-930.

Stack, J., Bostock, R., Hammerschmidt, R., Jones, J. and Luke, E. 2014. The National Plant Diagnostic Network: Partnering to protect plant systems. Plant Dis. 98:708-715.

Stack, J., Cardwell, K., Hammerschmidt, R., Byrne, J., Baldwin, W., Beck, H., Bostock, R., Thomas, C. and Luke, E. 2006. The National Plant Diagnostic Network. Plant Dis. 90:128-136.

Wilkes, M., Papageorgiou, S., Kim, T., Baldrias, L., Aguilar, E., Kelly, T., and Tee, M. 2019. One Health workers: Innovations in early detection of human, animal, and plant disease outbreaks. J. Global Health Rep. 3: e2019093. 